\title{
Caractéristiques de l'aviculture villageoise et influence des techniques d'amélioration sur ses performances zootechniques dans la province du Sourou, région Nord-Ouest Burkinabè
}

\author{
Bansé OUEDRAOGO ${ }^{1 *}$, Bayala BALE ${ }^{2}$, Sibiri Jean ZOUNDI ${ }^{1}$ et Laya SAWADOGO ${ }^{2}$ \\ ${ }^{1}$ Institut de l'Environnement et de Recherches Agricoles (INERA), \\ 04 BP 8645 Ouagadougou 04, Burkina Faso. \\ ${ }^{2}$ Université de Ouagadougou, UFR/SVT, 01 BP 7029 Ouagadougou 01Burkina Faso. \\ *Auteur correspondant, E-mail : banse_ouedraogo@yahoo.fr; Tél. : (+226) $50340270 / 50347112$
}

\section{RESUME}

Une caractérisation des systèmes d'élevage par des enquêtes combinées avec des suivis et mesures de paramètres zootechniques a été conduite dans dix huit (18) villages et un échantillon de (36) aviculteurs de la province du Sourou. Le but était aussi d'évaluer l'influence des techniques d'amélioration sur les performances zootechniques. Le système d'élevage est extensif, quelquefois semi-intensif. L'étude révèle des contraintes d'ordre sanitaire, alimentaire, d'habitat des volailles. Le suivi montre que les sujets en claustration ont une croissance plus élevée $(\mathrm{P}<0,05)$ à 16 semaines puisque les poids vifs ont atteint $1232 \mathrm{~g} /$ sujet en mode divagant et $1689 \mathrm{~g} /$ sujet en mode claustration. Les mortalités de 8,02\% montrent une probabilité de survie numériquement meilleure en comparaison avec les sujets en divagation (18,50\%). Les performances zootechniques restent relativement bonnes au regard des conditions de production des différents modes d'élevage. Une amélioration de la productivité s'impose et attention particulière sera accordée à une alimentation utilisant des ressources alimentaires locales, une conduite de l'élevage incluant un accès aux soins vétérinaires et un habitat adéquat. L'amélioration génétique des races locales par l'introduction de coqs améliorateurs est à renforcer.

(C) 2015 International Formulae Group. All rights reserved.

Mots clé : Burkina Faso, poulets, systèmes d'élevage, performances zootechniques.

\section{INTRODUCTION}

L'élevage représente la deuxième ressource du secteur primaire au Burkina Faso, avec une contribution de 15\% au PIB (MRA, 2006). Il reste la première source de revenus monétaires des ménages ruraux et leur permet ainsi, d'accéder aux services sociaux de base (MRAH, 2013). En 2012, l'effectif du cheptel national se chiffre à 8737 822 têtes de bovins, 8745273 ovins, 13094 133 caprins, 2254822 porcins, 1114676 asins, 39718 équins, 17659 camelins, 41674 321 volailles.

De par le passé, les décideurs politiques ont beaucoup misé sur les ruminants domestiques pour faire face à la demande croissante en produits carnés des populations. Malheureusement, face aux faibles résultats atteints, les stratégies se sont réorientées vers l'amélioration des animaux à cycle court, notamment les volailles (MRA, 2010). 
En effet, l'aviculture, part la plus importante des effectifs du cheptel burkinabè, occupe une place importante dans les moyens d'existence des populations surtout en milieu rural où les volailles sont élevées non seulement pour la consommation locale, les pratiques rituelles, coutumières, religieuses et sociales, mais aussi pour la génération de revenus à travers la vente (Pousga, 2009). C'est l'activité agricole la plus pratiquée tant par les hommes, les femmes que les enfants. Il se monnaye aisément sur les marchés locaux et procure des revenus substantiels (MRA, 2011). En dépit de sa place vitale dans la vie des populations en milieu rural, les performances de production de l'aviculture traditionnelle demeurent faibles et ses caractéristiques encore pas suffisamment connues.

En effet, les quelques rares travaux de recherche sur la caractérisation des systèmes avicoles villageois ont généralement concerné la zone sahélienne Nord du pays (Bonkoungou, 2005). D'autres travaux de diagnostic général de l'aviculture ont été menés (Ouédraogo, 2002; Pousga, 2009 ; Kondombo, 2007), mais beaucoup de zones sont restées en marge dont celle du Nordouest du pays ; espace géographique occupant la troisième place avec $12,30 \%$ des effectifs totaux de volaille du pays (DGPSE, 2011). Certains travaux n'ont abordé que des segments spécifiques comme le système de commercialisation (CEFCOD, 2013).

Tous ces travaux de recherche soulignent la nécessité de concentrer les efforts de développement sur la santé, l'alimentation, l'habitat ou l'amélioration génétique. Ces résultats soulignent également la nécessité d'entreprendre des travaux plus approfondis de caractérisation des performances et potentiels de ces élevages avicoles villageois afin de mieux cibler les actions d'amélioration, mais aussi de constituer un référentiel d'indicateurs de base pour la mesure d'impact.

La présente étude, conduite au NordOuest du Burkina Faso, dans le cadre de la recherche-accompagnement au projet
Millenium Challenge Account (MCA), est une contribution à cette demande du développement. Elle s'est fixée un objectif double, d'une part de faire un diagnostic de l'aviculture traditionnelle en vue de déceler les principales contraintes freinant son développement, et d'autre part, d'évaluer l'efficacité technique de paquets technologiques promus et vulgarisés dans le cadre du projet sur les performances zootechniques des volailles en comparant les performances observées pour les sujets élevés en claustration et ceux laissés en divagation. Les résultats et recommandations issus du travail devraient nourrir la réflexion et l'action sur l'amélioration des performances technicoéconomiques des systèmes avicoles traditionnels villageois.

\section{MATERIEL ET METHODES \\ Matériel \\ Description du site}

L'étude a été conduite dans la province du Sourou au Nord-Ouest du Burkina Faso. La province du Sourou a une latitude de $13.0667^{\circ}$ Nord et une longitude de $3.0667^{\circ}$ Ouest. Elle est limitée au Nord par la province du Yatenga et la République du Mali, au Sud par les provinces du Mouhoun et du Sanguié, à l'Est par les provinces du Yatenga, du Passoré et à l'Ouest par les provinces du Mouhoun et de la Kossi. Cette province connaît l'alternance d'une saison sèche et d'une saison pluvieuse. Elle se divise en 2 sous zones climatiques dont la sous-zone soudanosahélienne couvrant la partie Nord de la province. Les précipitations s'étalent sur une période de 3 mois et les hauteurs d'eau moyennes recueillies par an varient entre 300 et $700 \mathrm{~mm}$. La deuxième sous-zone soudanienne occupe la partie sud de la province et se caractérise par des hauteurs d'eau annuelles de 600 à 800 mm sur quatre mois de pluies (Figure 1).

\section{Méthodes \\ L'échantillonnage}

Trente-six (36) aviculteurs ont été enquêtés dans dix-huit (18) villages soient 2 
producteurs par village. A partir de cet échantillon de base, un deuxième échantillon de six (6) aviculteurs dits «leadeurs » sélectionnés à partir des Fermes d'Elevage Modèle (FEM), et disposant d'au moins 50 têtes chacun, a été identifié pour le suivi rapproché des élevages en claustration. La sélection des aviculteurs a été faite à travers des consultations avec les services techniques et auxiliaires d'élevage et sur la base de critères précis tels l'effectif du cheptel volaille, l'habitat, etc.

\section{Conduite des enquêtes et suivi des élevages}

Les interviews ont été réalisées à l'aide d'un guide d'entretien. Les informations collectées concernent essentiellement la conduite technique, la santé, l'habitat, la productivité des poules, les prix des produits avicoles, l'alimentation, les performances zootechniques, les principales contraintes de développement. Le suivi a duré de novembre 2013 à février 2014.

\section{Évaluation de paquets technologiques promus dans le cadre du projet}

Le matériel animal est constitué de 80 poussins hybrides issus d'un croisement entre des coqs ISA Brown et des poules de race locale repartis en deux lots pour l'expérimentation (Tableau 1).

Les gains de poids et l'indice de consommation ont été évalués sur une période de 120 jours. Les animaux sont soumis à un programme de prophylaxie. Deux types de rations correspondant à deux phases de l'élevage ont été utilisés. Une ration pour la phase de démarrage et une autre pour la croissance -finition (Tableau 2).

\section{Analyse statistique}

Les données ont été organisées à l'aide du logiciel Excel 2000 de Microsoft Office. Les analyses des données qualitatives et la comparaison des moyennes ont été effectuées par le test d'analyse de variance (ANOVA) à deux niveaux (système « semi intensif » et système « divagant) à l'aide du logiciel Statistical Package for the Social Science (SPSS) version 2.0. En cas de différence significative, la classification des moyennes a été obtenue par le test de Newman-Keuls au seuil de $5 \%$.

\section{RESULTATS}

Caractéristiques générales des élevages avicoles villageois

\section{Composition des élevages}

Les espèces aviaires élevées sont le poulet, la pintade, le canard en général et accessoirement les pigeons. La taille moyenne des poulets est pour l'ensemble des villages cibles de 58 poulets par exploitation avec $39 \%$ de poussins, $31 \%$ de poulettes et coquelets, $25 \%$ de poules et $5 \%$ de coqs. Le rapport coq/poules est de 1 coq pour 4 poules en moyenne par aviculteur. La poule est l'espèce la plus répandue car toutes les exploitations en possèdent. Quant aux pintades, l'effectif moyen par exploitation est de 8 sujets. Le canard est beaucoup moins répandu que les autres espèces; seuls $22,5 \%$ des aviculteurs en possèdent.

\section{Caractéristiques des systèmes de production}

L'élevage en liberté représente $56,5 \%$ de cas dans la zone contre $38,2 \%$ pour le système en semi-liberté et $5,3 \%$ en claustration total.

\section{Système avicole traditionnel de type extensif \\ C'est un système traditionnel où les} élevages sont essentiellement un mélange d'espèces et d'âges différents puis gérés avec des pratiques rudimentaires de conduite sanitaire, d'habitat, d'alimentation, d'abreuvement. Ces élevages se caractérisent par un faible investissement financier et matériel connaissant de fortes mortalités. Ce sont des exploitations familiales réparties en petites unités de production comportant en moyenne 5 à une cinquantaine de têtes chacune. Ce type d'élevage dont les normes rationnelles de conduite d'élevage sont reléguées au second plan, assure rarement la totalité des besoins des éleveurs du fait de la précarité des conditions de vie des oiseaux, notamment l'absence d'habitat adéquat, l'insuffisance ou absence d'alimentation équilibrée, la prédation et les problèmes 
sanitaires. L'habitat comprend des logements précaires mal aérés et généralement pas bien entretenus et nettoyés. Ces poulaillers sont rudimentaires et les animaux vivent la plupart du temps dans la cour et souvent autour des concessions (Figure 2).

Plus de $54 \%$ des éleveurs enquêtés utilisent des mangeoires traditionnelles. Les abreuvoirs sont parfois un morceau de canaris placé quelque part dans la concession (Figure $3)$.

Les éleveurs alimentent leurs volailles essentiellement avec l'eau de forage (35\%) et les puits $(42 \%)$. Les autres sources d'abreuvements sont les barrages, puisards, etc. Il n'existe pas à proprement parler de mangeoires, ni d'abreuvoirs mais des récipients de fortune. La non utilisation de ces équipements d'élevage par les aviculteurs villageois est principalement due à une méconnaissance $(79 \%)$ mais aussi au manque de moyens financiers (21\%). L'absence de réelle stratégie de gestion d'exploitation des animaux fait que les animaux en âge d'être exploités commercialement le sont peu car très souvent, les ventes se font en fonction des besoins de la famille ou des opportunités de profit maximum à l'approche des périodes de fêtes. Les circuits commerciaux sont complexes car les producteurs vendent à la fois aux grossistes, aux détaillants et aux consommateurs.

\section{Santé des volailles}

$\mathrm{La}$ couverture prophylactique et thérapeutique est quasi inexistante avec comme conséquence la persistance de pathologies infectieuses telles que les maladies de Newcastle, la bronchite infectieuse, le choléra, la variole aviaire et des maladies parasitaires comme les coccidioses. Les éleveurs $(12 \%$ des cas) ont essentiellement recours à la pharmacopée traditionnelle à l'aide des extraits de feuilles ou d'écorce. Le suivi sanitaire des poules est globalement précaire et le taux de mortalité estimé est de $77,8 \%$. En général, on enregistre, à l'âge de 0-3 mois, le plus fort taux de mortalité des poussins (58\%), ce qui contribue à réduire la productivité. Cette forte mortalité des poulets n'incombe pas seulement aux maladies, mais aussi à d'autres facteurs climatiques en lien avec les modes d'élevage. Les maladies sévissent surtout en saison sèche chaude dans ( $29 \%$ des cas), en hivernage ( $16 \%$ des cas) et en saison sèche froide (40\%); seulement 14\% des répondants estiment qu'elles sévissent à tout moment.

\section{Espèces élevées}

La quasi-totalité des producteurs élèvent des races locales et la poule locale représente 63\% des effectifs sur l'ensemble de la zone. Elle est de grande taille, d'un poids moyen de $1,25 \mathrm{~kg}$. Les pintades représentent $30,7 \%$ des volailles élevées; Elles sont de grande taille, avec un poids variant de $1,5 \mathrm{~kg}$ à $2 \mathrm{~kg}$. Les autres volailles (dindons, pigeons, canards) représentent $6,3 \%$ des effectifs.

\section{Objectif de l'exploitation des animaux}

Les principaux objectifs sont la vente, les sacrifices, la production d'œufs, l'autoconsommation, etc. Les produits avicoles sont destinés à la vente pour $69 \%$ des cas, les dons $18,2 \%$, et l'autoconsommation $13,3 \%$. Les objectifs sont moyennement atteints dans la zone. Les revenus de la vente sont destinés aux dépenses familiales pour une part non négligeable.

\section{Conduite de l'alimentation avicole}

L'alimentation repose essentiellement sur la divagation chez la plupart des producteurs. Les volailles sont en liberté et trouvent elles-mêmes leur nourriture, notamment les vers, feuilles, termites, résidus de cuisine, etc. Quelques rares fois, elles reçoivent du mil, du sorgho, du son, dont une poignée est jetée le matin et rarement le soir (Tableau 1). Les pintades et canards selon leur âge sont soumis au même mode d'alimentation que les poulets. Toutes les espèces aviaires sont mélangées et s'alimentent de la même manière.

\section{Système d'aviculture traditionnelle améliorée \\ Ce système concerne les exploitations de taille relativement plus importante, de l'ordre de 30 têtes à plus d'une centaine de sujets. En plus de sa fonction socioculturelle}


et nutritionnelle, ce système intègre un objectif économique relativement important à travers la vente des oiseaux vivants dans les marchés locaux. On y rencontre des poulaillers simples construits en matériaux locaux plus résistants, de dimensions variables et de qualité plus ou moins acceptable (Figure 4) suivant les moyens financiers de l'éleveur, les objectifs et la taille de l'élevage. Les équipements (abreuvoir) sont pour la plupart à base de matériaux de récupération. Le modèle vulgarisé dans la zone est généralement un canari en argile cuite (Figure 5).

Les éleveurs alimentent leurs volailles avec l'eau de forage (35\%), les puits (42\%). Dans les villages bénéficiant de l'encadrement du projet, l'élevage des poules est tenu par les femmes à $86,6 \%$. A l'instar des élevages modernes, la gestion d'exploitation intègre le ciblage de la période favorable de vente avec parfois la production d'un type précis de produit pour un marché particulier (jeune pintade à griller, dindons pour les fêtes de fin d'année, etc.)

\section{Espèces élevées}

Les producteurs élèvent généralement des animaux issus du croisement avec les coqs raceurs introduits par le projet $(96 \%$ des effectifs sur l'ensemble de la zone). Les espèces élevées sont en majorité des poulets et le poids moyen est de $1,50 \mathrm{~kg}$. Les pintades représentent $3 \%$ des volailles élevées, avec un poids moyen variant de $1,5 \mathrm{~kg}$ à $2 \mathrm{~kg}$. Les autres volailles représentent $1 \%$ des effectifs.

\section{Objectifs de production}

L'essentiel des produits avicoles est destiné à la vente $(80 \%)$, la production d'œufs pour le renouvellement des effectifs (17\% des cas), l'autoconsommation (2\%) et les autres évènements (1\%). Dans 65\% des cas, les objectifs sont atteints dans la zone et les ventes sont effectuées dans les marchés des grands centres urbains ( $90 \%$ des cas). Les revenus de la vente sont destinés aux dépenses familiales ( $75 \%$ des cas). Ce système a bénéficié d'opportunité de micro-crédit (95\% des producteurs) à travers des organismes spécialisés de microfinance.

\section{Conduite de l'alimentation}

Deux modes d'alimentation sont rencontrées, celui des jeunes et celui des adultes. Les poussins reçoivent des sons de céréales, des céréales (mil, sorgho, maïs), des os brûlés, de l'arachide, des termites, des asticots, etc. Poussins et mères poules sont élevés ensemble en semi-claustration. Les quantités distribuées varient selon les moyens de l'aviculteur, la saison et l'importance des effectifs (Tableau 2).

\section{Suivi sanitaire de la volaille}

On note l'existence de programmes de vaccination contre les maladies infectieuses dominantes avec de bons résultats, notamment les faibles taux de mortalité $(25 \%)$ pour les animaux en semi-claustration ou en claustration permanente. Dans les villages d'intervention du projet, $85 \%$ des ménages interrogés utilisent des médicaments vétérinaires.

\section{Suivi des paramètres de production \\ Paramètres de reproduction}

L'âge à la maturité sexuelle est d'environ 5 mois pour le coq, de 6 mois pour la poule. En termes de performances de ponte, le suivi révèle que les volailles locales sont de mauvaises pondeuses. Chez la poule, la production d'œufs dure toute l'année et elle pond en moyenne 5 à 12 œufs par cycle de ponte, trois à quatre fois dans l'année. L'incubation dure 21 jours et la période de ponte maximale est la saison sèche froide. Le nombre moyen d'œufs couvés par les femelles a été de 13 œufs. Le poids moyen des œufs des poules est de $38 \mathrm{~g}$. Le taux global d'éclosion enregistré a été de $65 \%$.

\section{Performances de croissance}

Evolution pondérale : L'évolution
pondérale présente des variations
significatives jusqu'à la fin de la $16^{\mathrm{e}}$ semaine
selon le mode d'élevage. Aucune différence
significative n'est observée en ce qui concerne
le poids à l'éclosion qui est de $38 \mathrm{~g}$ en
moyenne. En mode divagant, les animaux se
nourrissaient de feuilles, de graines de
végétaux divers, des graines de céréales, ainsi
que d'insectes, de vers, etc. Pour les poulets


en élevage libre, le poids moyen à la fin de la phase de démarrage (1 mois) a été de $319 \mathrm{~g}$; ce poids est passé à 1232 g à 4 mois d'âge. La moyenne générale à la fin de la phase de démarrage est de $366 \pm 6 \mathrm{~g}$ par poulet. Pour les sujets en claustration on est passé de $434 \mathrm{~g}$ en fin démarrage à $1689 \mathrm{~g}$, à 4 mois d'âge. La moyenne générale à la fin de la phase de démarrage dans ce cas est de $685 \pm 6$ g par poulet. La différence de poids entre les différents lots de poulets est statistiquement significative quelle que soit la période d'élevage. Au terme de l'essai (120 jours), les poulets pesaient $1689 \mathrm{~g}$ par sujet en claustration contre $1232 \mathrm{~g}$ pour les témoins en élevage libre. L'âge de commercialisation était atteint à partir de 3 à 4 mois (Tableau 4).

- Gain moyen quotidien (GMQ) : Le GMQ pendant la phase de démarrage (1 à 30 jours) du lot d'animaux en claustration était de $16 \pm$ $3 \mathrm{~g} / \mathrm{j}$; celui des animaux en élevage libre étant de $14 \pm 3 \mathrm{~g} / \mathrm{j}$. Sur l'ensemble de la période de l'essai $(120 \mathrm{j})$, des variations significatives $(\mathrm{P}<0,05)$ ont été observées au niveau des GMQ, $27 \pm 4$ g pour les animaux en claustration, contre $19 \pm 4 \mathrm{~g}$ pour les animaux en élevage libre. D'une manière générale, les poulets en claustration présentent les meilleurs GMQ sur l'ensemble de la période.

- Indice de consommation (IC) : Les valeurs de l'indice de consommation au démarrage étaient de 1,82 pour le lot en claustration. Il est passé à 1,98 entre 1 et 2 mois et à 2,30 entre 1 et 3 mois d'âge. Sur l'ensemble de la période d'élevage (120 j), l'IC des poulets en claustration était de 2,02 .

Les taux de mortalité : Le taux de mortalité globale a été de $10,15 \%$ pour l'ensemble des lots. Les poulets en élevage libre ont enregistré le taux le plus élevé (18,50\%); celui des animaux en claustration ayant été de $8,02 \%$.

\section{Données économiques}

L'aviculture traditionnelle requiert peu d'investissement financier comparée à celle traditionnelle améliorée.
En mode claustration, les principales dépenses effectuées sont liées à l'alimentation et les soins vétérinaires. Les volailles sont vendues en moyenne à 3000 FCFA en élevage traditionnel amélioré. Le producteur peut vendre au moins 15 poulets par mois avec une marge nette d'environ de 34835 FCFA /mois pour un cycle de 3 mois.

En mode divagant, l'achat d'aliment est faible (juste de la complémentation) avec les traitements et prophylaxie faisant appel à la pharmacopée traditionnelle. L'âge d'abattage des poulets est tardif et dépend, en fait, du poids des animaux. Le producteur peut vendre au moins 6 poulets par mois à un prix unitaire moyen de 2000 FCFA avec une marge nette de 9950 FCFA/mois en moyenne pour un cycle de 5 mois. La rentabilité des élevages reste relativement bonne selon les différents modes d'élevage. Les recettes générées sont utilisées pour assurer les dépenses domestiques, la scolarisation des enfants, le remboursement de crédit, l'épargne. Pour certains producteurs, l'argent sert également aux achats de denrées alimentaires et d'autres pour le renouvellement du cheptel.

\section{Principales contraintes}

Les principales contraintes varient selon les types d'élevage.

En système extensif, les principales contraintes sont les maladies, l'habitat inadéquat et les problèmes alimentaires, avec comme conséquence les fortes mortalités enregistrées. Dans la plupart des cas, l'absence de bons géniteurs constitue également une contrainte majeure à l'amélioration de la productivité des élevages.

En système traditionnel amélioré, les principales contraintes demeurent la technicité des aviculteurs, notamment la faible connaissance des techniques de formulation des aliments et de rationnement en fonction des stades physiologique. A cela s'ajoute les coûts élevés des ingrédients entrant dans la production des aliments, ainsi que les difficultés d'accès à des services vétérinaires de proximité. 


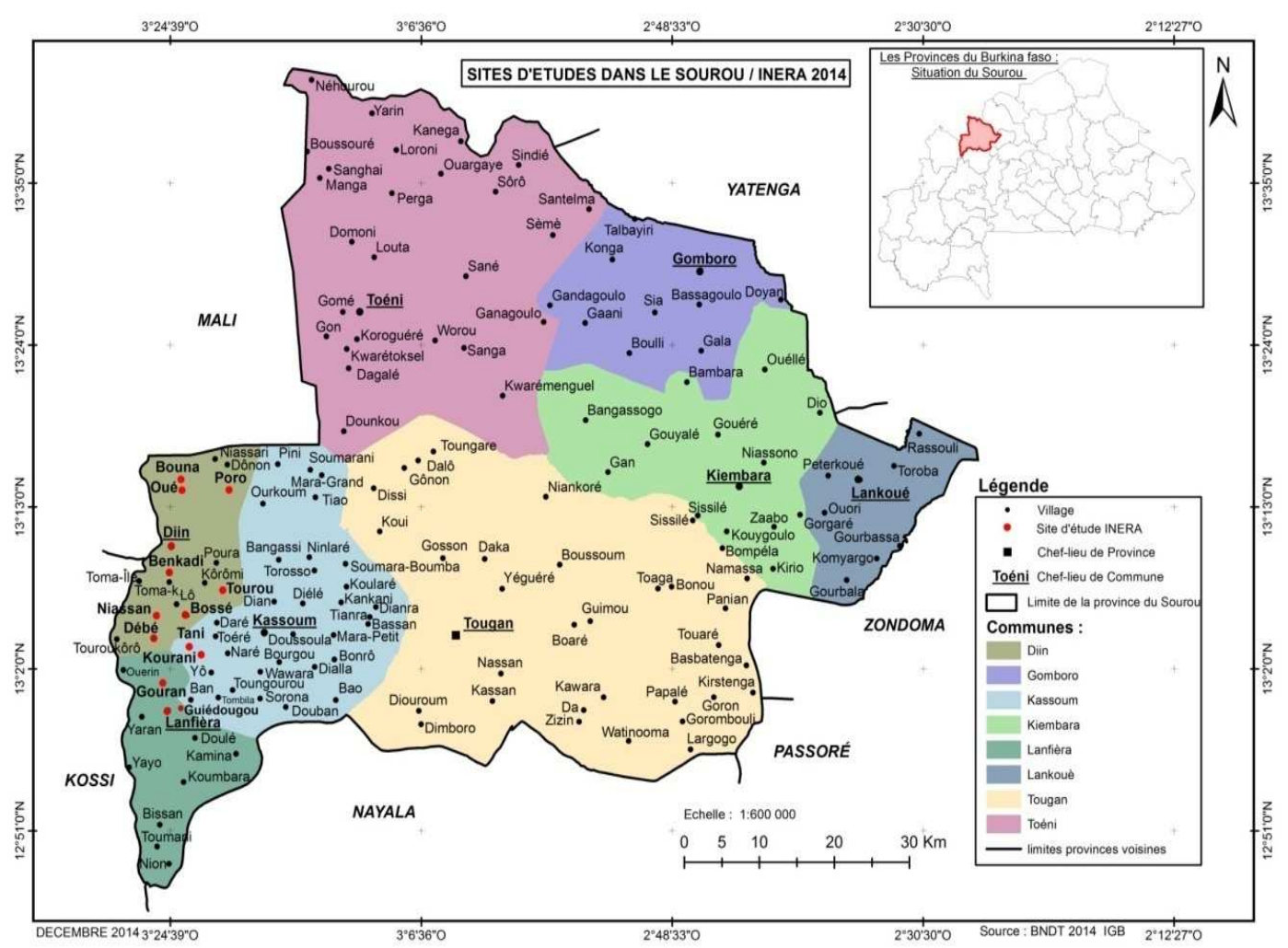

Figure 1: Localisation de la province du Sourou sur la carte du Burkina Faso. IGB 2014.

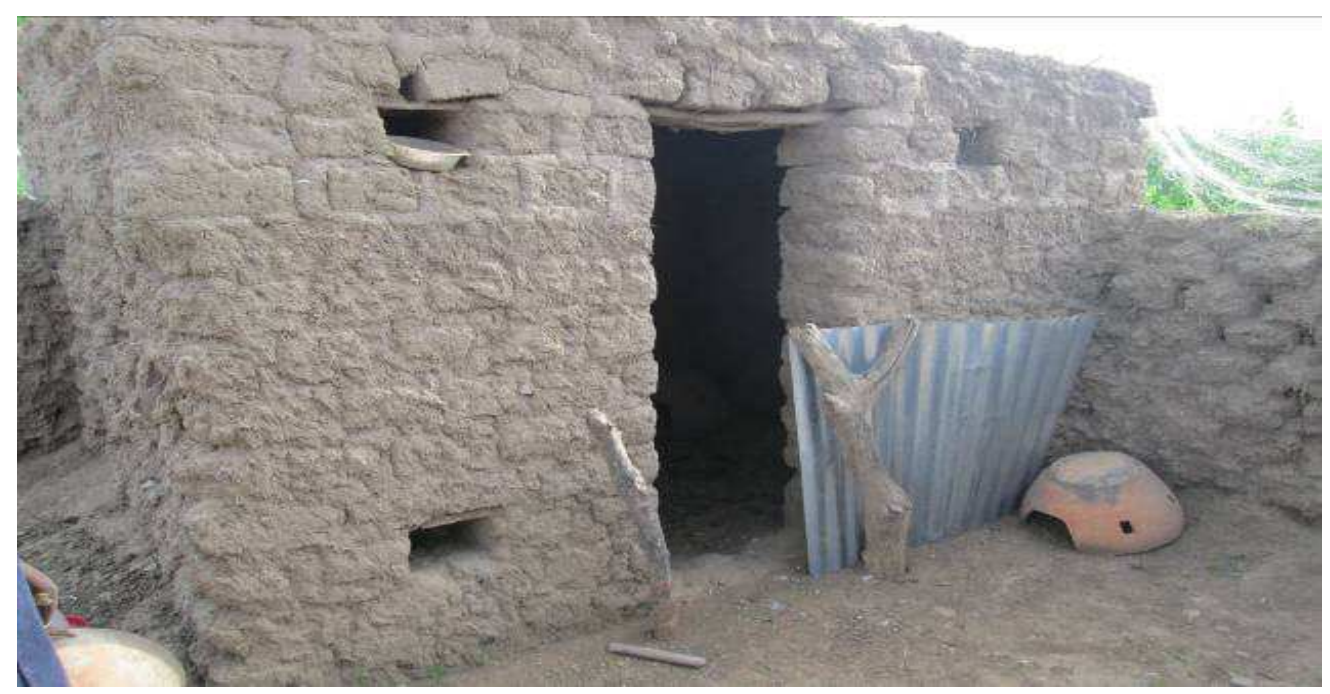

Figure 2 : Habitat de volaille chez un producteur non encadré (village de Tani). 
B. OUEDRAOGO et al. / Int. J. Biol. Chem. Sci. 9(3): 1528-1543, 2015

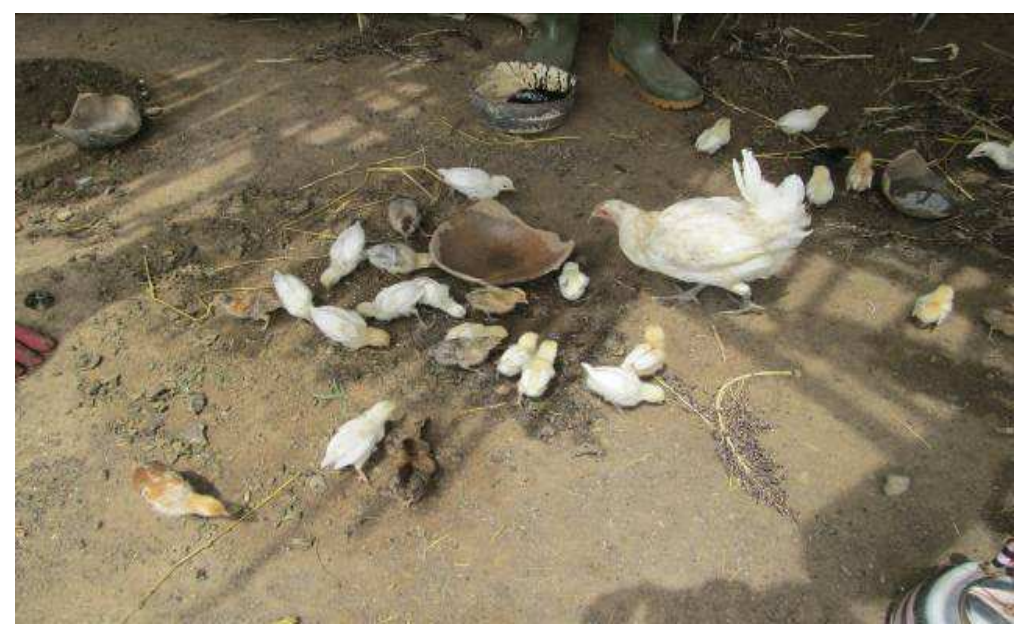

Figure 3: Morceau de canari servant d'abreuvoir.

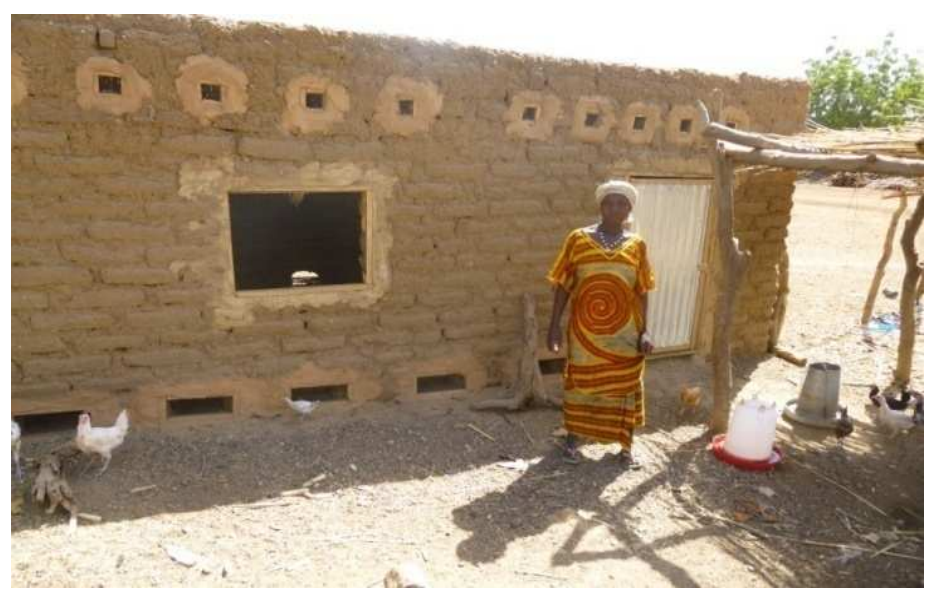

Figure 4: Productrice devant son poulailler amélioré à Oué.

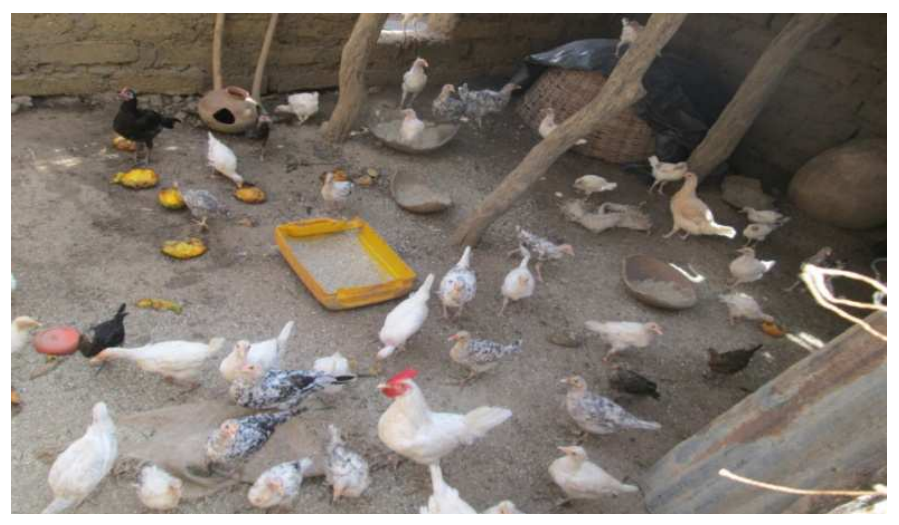

Figure 5: Modèle d'abreuvoir semi-amélioré et mangeoire en matériel de récupération. 
Tableau 1 : Mise en lot et Traitements appliqués.

\begin{tabular}{|c|c|c|c|c|c|}
\hline & \multicolumn{5}{|c|}{ Traitements appliqués } \\
\hline & \multirow{2}{*}{$\begin{array}{l}\text { Périodes } \\
\text { d'élevage }\end{array}$} & \multicolumn{2}{|c|}{ Divagation } & \multicolumn{2}{|c|}{ Claustration } \\
\hline & & Sous-lot 1 & Sous-lot 2 & Sous-lot 1 & Sous-lot 2 \\
\hline $\begin{array}{l}\text { Nombre } \\
\text { d'animaux }\end{array}$ & & 20 & 20 & 20 & 20 \\
\hline \multirow[t]{2}{*}{$\begin{array}{l}\text { Conduite de } \\
\text { l'alimentation }\end{array}$} & démarrage & $\begin{array}{l}\text { Divagation avec } \\
\text { complément en } \\
\text { son de céréale, } \\
\text { brisures de riz, } \\
\text { résidus de cuisine }\end{array}$ & $\begin{array}{l}\text { Divagation avec } \\
\text { complément en } \\
\text { son de céréale, } \\
\text { brisures de riz, } \\
\text { résidus de } \\
\text { cuisine }\end{array}$ & $\begin{array}{c}\text { 60g/j/sujet de } \\
\text { l'aliment } \\
\text { poussin } \\
\text { (Tableau 2) }\end{array}$ & $\begin{array}{c}60 \mathrm{~g} / \mathrm{j} / \mathrm{sujet} \\
\text { de l'aliment } \\
\text { poussin } \\
\text { (Tableau 2) }\end{array}$ \\
\hline & $\begin{array}{l}\text { croissance } \\
\text {-finition }\end{array}$ & $\begin{array}{l}\text { Céréales, feuilles ; } \\
\text { grain d'herbe ; } \\
\text { termite ; asticot } \\
\text { résidu de cuisine }\end{array}$ & $\begin{array}{l}\text { Feuilles ; grain } \\
\text { d'herbe ; } \\
\text { termite ; asticot } \\
\text { résidu de cuisine }\end{array}$ & $\begin{array}{c}90 \mathrm{~g} / \mathrm{j} / \text { sujet de } \\
\text { l'aliment } \\
\text { croissance - } \\
\text { finition } \\
\text { (Tableau 2) } \\
\end{array}$ & $\begin{array}{c}\text { 90g/j/sujet } \\
\text { de l'aliment } \\
\text { croissance - } \\
\text { finition } \\
\text { (Tableau 2) }\end{array}$ \\
\hline habitat & & \multicolumn{2}{|c|}{ poulaillers rudimentaires. } & \multicolumn{2}{|c|}{$\begin{array}{l}\text { Habitat amélioré en matériaux } \\
\text { locaux. }\end{array}$} \\
\hline santé & & \multicolumn{2}{|c|}{ recours à la pharmacopée traditionnelle } & \multicolumn{2}{|c|}{$\begin{array}{l}\text { Existence d'un programme de } \\
\text { vaccination }\end{array}$} \\
\hline
\end{tabular}

Tableau 2 : Composition des aliments fabriqués à la ferme.

\begin{tabular}{lcc}
\hline Ingrédients $(\%)$ & Aliment démarrage & Aliment croissance -finition \\
\hline Maïs & 59 & 58 \\
Son de sorgho & 16,5 & 19 \\
Tourteaux d'arachide & 7 & 4 \\
Tourteaux (coton) & 3 & 7 \\
Farine de poisson & 9 & 6,5 \\
Poudre d'os brûlé & 2,5 & 2,5 \\
Sel de cuisine & 0,5 & 0,5 \\
CMV & 2,5 & 2,5 \\
Total & $\mathbf{1 0 0}$ & $\mathbf{1 0 0}$ \\
\hline CMV : Complexe Minéral Vitaminique & &
\end{tabular}

CMV : Complexe Minéral Vitaminique 
Tableau 3 : Caractéristiques des deux systèmes d'élevage analysés.

\begin{tabular}{|c|c|c|c|c|c|}
\hline & Gestion du troupeau & Importance destination & Race et habitat & $\begin{array}{c}\text { Mortalités santé et } \\
\text { alimentation }\end{array}$ & $\begin{array}{c}\text { Production } \\
\text { couvaison }\end{array}$ \\
\hline Système traditionnel & $\begin{array}{c}\text { Familiale très majoritaire } \\
\text { Entretien en majorité par } \\
\text { les femmes et enfants }\end{array}$ & $\begin{array}{c}\text { Consommation } \\
\text { domestique } \\
\text { Faible revenu } \\
\text { Importance sociale et } \\
\text { Culturelle }\end{array}$ & $\begin{array}{c}\text { Races locales } \\
\text { Pas d'habitat ou habitat } \\
\text { très sommaire }\end{array}$ & $\begin{array}{c}\text { Forte mortalité de l'ordre de } \\
60 \% \\
\text { Pas de programme } \\
\text { d'alimentation } \\
\text { (divagation) } \\
\text { Pas de vaccination } \\
\text { traitements traditionnel }\end{array}$ & $\begin{array}{c}30 \text { à } 50 \\
\text { oeufs/an/poule } \\
\text { Longues périodes } \\
\text { de } \\
\text { couvaison } \\
\text { Croissance lente }\end{array}$ \\
\hline $\begin{array}{l}\text { Système traditionnel } \\
\text { amélioré }\end{array}$ & $\begin{array}{c}\text { familiale } \\
\text { limitée au propriétaire } \\
\text { Entretien par les femmes } \\
\text { et hommes }\end{array}$ & $\begin{array}{c}\text { Consommation } \\
\text { domestique } \\
\text { et vente dans les marchés } \\
\text { locaux } \\
\text { Revenu familial } \\
\text { Importance sociale } \\
\text { Nécessite financement par } \\
\text { Micro-crédit }\end{array}$ & $\begin{array}{c}\text { Races } \\
\text { locales/améliorées } \\
\text { Hybrides (poulets de } \\
\text { chair ou } \\
\text { pondeuses) } \\
\text { Habitat simple ou } \\
\text { habitat avec des cages } \\
\text { etlitières }\end{array}$ & $\begin{array}{c}\text { Mortalité faible autour de } 35 \% \\
\text { Alimentation locale (semi- } \\
\text { divagation) } \\
\text { Alimentation équilibrée } \\
\text { Vaccination contre la maladie } \\
\text { de Newcastle } \\
\text { Schémas de } \\
\text { vaccination } \\
\text { Traitement régulier }\end{array}$ & $\begin{array}{c}50 a ̀ ~ 250 \\
\text { oeufs/an/poule } \\
\text { Courtes périodes } \\
\text { de couvaison } \\
\text { Croissance rapide }\end{array}$ \\
\hline
\end{tabular}


Tableau 4 : Evolution pondérale des différents lots de poulets de chair (en g).

\begin{tabular}{|c|c|c|c|c|c|}
\hline \multirow[t]{2}{*}{ Ages } & \multirow[t]{2}{*}{ claustration } & \multirow[t]{2}{*}{ Mode divagant } & Moyenne & ET & valeur \\
\hline & & & & & $\mathbf{P}$ \\
\hline 1 semaine & 38 & 38 & 38 & 1 & 0,975 \\
\hline 4 semaines & 434 & 319 & 366 & 6 & 0,000 \\
\hline 8 semaines & 921 & 621 & 685 & 154 & 0,000 \\
\hline 16semaines & 1689 & 1232 & 1368 & 233 & 0,000 \\
\hline
\end{tabular}

\section{DISCUSSION}

Caractéristiques générales des élevages

Quel que soit le mode d'élevage ou le type d'espèce élevé, l'enquête révèle de façon générale que les propriétaires des élevages sont en majorité (79\% des cas) des hommes. Cependant, l'élevage avicole est essentiellement l'œuvre des femmes ( $82 \%$ des cas). Ces résultats confirment ceux obtenus par Ouédraogo (2000) et Pousga (2009) qui indiquent que l'aviculture en milieu villageois ou familial est surtout une activité des femmes et des enfants. Au-delà de cette tendance générale, nos résultats indiquent des exceptions dans certains villages où l'élevage est pratiqué au même degré par les hommes que par les femmes, contrairement à ce qui se passe dans d'autres pays où l'activité est essentiellement féminine (Moussa et al., 2010).

\section{Caractéristiques du système extensif Conduite alimentaire des animaux}

Le système d'alimentation courant des animaux repose sur la divagation. Les volailles sont élevées en liberté et leur alimentation est essentiellement constituée d'aliments résiduels «picorables». Nos résultats corroborent ceux obtenus dans d'autres pays (Sonaiya et al., 2004 ; Ayssiwede et al., 2011). Les aliments comprennent des végétaux (feuilles, fleurs, etc.), des insectes, des grains ou de sons de céréales autour des mortiers ou servis par l'aviculteur, etc. Des résultats similaires ont été observés dans d'autres zones du Burkina Faso (Pousga 2009) et confirmé par les travaux de (Goromela et al., 2006). Ces difficiles conditions d'alimentation expliqueraient les faibles rendements observés dans ce système de production.

\section{L'habitat}

Il n'y a pratiquement pas d'habitat approprié assurant une bonne protection des oiseaux face aux intempéries et aux prédateurs. Dans la plupart des cas, des abris rudimentaires ou poulaillers sommaires construits sans aucune norme à base de matériaux locaux (tiges et pailles de graminées, débris de tôles, etc.) sont utilisés, comme le souligne (Diagne, 2012). Nos observations sont également en accord avec celles faites par Pousga (2009) ainsi que Nmoula et al. (2012) qui indiquent qu'en milieu rural, $80 \%$ des poulaillers sont construits selon le style traditionnel avec du banco (73\% des poulaillers) ou de la paille (7\% des poulaillers). Ils soulignent par ailleurs que dans certains cas, les animaux ne bénéficient d'aucun abri et passent la nuit perchés sur les arbres. Cette situation les expose davantage à la prédation et aux intempéries diverses, principales causes des fortes mortalités observées. Des travaux similaires sur l'habitat de la volaille traditionnelle (Kondombo, 2007) montrent que ces abris sommaires à base de matériaux locaux sont par ailleurs mal entretenus, non désinfectés, rarement nettoyés; leurs toitures servant généralement de dépotoirs d'objets inutiles de la maison.

\section{Suivi sanitaire des animaux}

L'aviculture traditionnelle en Afrique sahélienne connaît une mortalité élevée surtout chez les poussins et les pintadeaux comme l'a fait remarquer plusieurs auteurs 
(Ouedraogo, 2002; Sangaré, 2005) avec des taux de mortalité atteignant 43 à $63 \%$ chez les jeunes au Sénégal. Les résultats de la présente étude indiquent que la mortalité des jeunes sujets est particulièrement élevée (80\%) entre la naissance et 3 mois d'âge. La maladie couramment rencontrée est la pseudo peste aviaire ou maladie du Newcastle. Cette maladie qui sévit généralement au mois de juin sous forme épizootique peut décimer jusqu'à $80 \%$ du cheptel et ceci conforte les informations données par El-Yuguda, (2009). Il convient de souligner que le mélange d'animaux d'âges différents, élevés ensemble dans une promiscuité totale, constitue aussi un facteur rendant plus complexe et compliqué le suivi sanitaire. Ceci corrobore l'étude de Ndayisenga (2010) qui a trouvé que la contamination poly-microbienne associée à une déficience alimentaire quantitative et qualitative, est le point de départ de diverses affections aviaires rencontrées dans la plupart des pays d'Afrique.

\section{Caractéristiques du système traditionnel amélioré}

Les caractéristiques des élevages traditionnels améliorés sont identiques à ceux décrits dans d'autres pays (Mopate et al., 2010). On trouve ici à coté de la race locale, les races améliorées à viande, de race pure ou hybride qui sont des produits de croisement de 2 races pures. Ce système utilise les races améliorées mixtes qui ne sont spécialisées ni pour la ponte ni pour la chair, mais avec des performances pondérales relativement meilleures que les races locales. Des ménages urbains et ruraux sont impliqués dans ce système. La taille des bandes varie de quelques dizaines à une centaine de têtes. Les performances de croissance sont meilleures comparées au système traditionnel extensif ; l'abattage intervient entre 12 et 16 semaines d'âge avec un poids moyen de 1500 grammes. Ceci confirme les résultats de Awa et al. (2008) selon lesquels les poulets en élevage amélioré atteignent un poids vif de 1.5 à $2 \mathrm{~kg}$ entre 3 et 5 mois d'âge. Mais leurs désavantages est la faible résistance aux maladies, d'où l'exigence d'une hygiène adéquate et une alimentation équilibrée.

\section{Conduite de l'alimentation}

L'alimentation des sujets est assez régulière, plus rationnelle et relativement équilibrée. Ceci confirme les observations faites dans d'autres travaux antérieurs (Ayssiwede et al., 2011). Ils affirment en effet que l'aliment est fabriqué à base de ressources locales complétées par des déchets de cuisine, des insectes (termites, acariens ou asticots, chenilles, vers de terre).

\section{L'habitat}

Le projet a pu mettre en place des poulaillers type amélioré vulgarisé dans la zone. Il s'agit de poulaillers en ciment avec une ouverture en façade grillagée. D'autres travaux (Ayssiwede et al., 2011) en Gambie révélaient que seuls $10 \%$ des éleveurs disposaient de poulaillers acceptables. Au Burkina Faso, Ouedraogo (2010) et kondombo (2007) indiquaient une amélioration de la conduite par l'utilisation d'habitat amélioré car le sous-secteur a bénéficié du soutien de plusieurs programmes et projets au cours des cinq dernières années. Plus de $15 \%$ des aviculteurs villageois disposaient de poulaillers améliorés.

\section{Suivi sanitaire}

La faible mortalité des oiseaux $(08,02 \%)$ observée est similaire $(9.8 \%)$ à celle observée par d'autres travaux avec des animaux élevés en confinement en station (Diagne et al., 2012). Cela s'explique notamment par le fait que les volailles bénéficient d'un suivi sanitaire plus rigoureux.

\section{Performance de croissance}

Le poids moyens enregistré en fin de période est inférieur aux valeurs $(1423 \pm 198$ g) obtenues par (Diagne et al., 2012). Les performances de croissance supérieures des sujets élevés en confinement comparées au mode en divagation, s'expliqueraient en partie par l'alimentation plus adéquate et équilibrée. Toutefois, à une semaine d'âge, les animaux en confinement et ceux en élevage libre avaient sensiblement les mêmes poids vifs. Ces performances s'expliqueraient par le fait 
que les poussins en élevage libre ont été nourris avec des termites et des grains de céréales concassés (maïs) dans la première semaine de leur vie avant d'être livrés à la divagation. Des observations similaires ont été faites par d'autres études qui ont confirmé l'impact positif d'une alimentation consistante sur les performances de croissance des poulets (Ayssiwede et al., 2011). Ces résultats confortent l'assertion des mêmes auteurs selon laquelle la conduite des oiseaux en confinement dans des systèmes traditionnels améliorés peut améliorer les effets néfastes des conditions environnementales sur l'état de santé et le bien-être de ceux-ci.

\section{Contraintes et perspectives d'amélioration}

Les contraintes essentielles du soussecteur de l'aviculture familiale relevées par des études antérieures (MRA, 2007) et les entretiens avec des producteurs sont liées aux conditions rudimentaires d'élevage (habitat, alimentation, suivis sanitaires) ainsi que l'amélioration génétique.). En effet, l'amélioration de l'habitat, de l'alimentation, de l'état sanitaire des volailles en milieu rural constatée pendant l'étude ont été essentielles pour le développement de l'aviculture.

Face aux nombreuses contraintes, les pistes d'amélioration suggérées par l'étude concernent l'amélioration des itinéraires techniques, la formulation de la ration alimentaire en utilisant des ressources alimentaires locales afin de réduire les coûts de production (utilisation par exemple des résidus de maraîchage, des termites, des asticots). S'agissant de l'amélioration de soins sanitaires, il faudrait l'étendre au contrôle des pathologies et la réduction du coût de traitement des maladies les plus rependues. Un contrôle sanitaire rigoureux s'impose par la lutte contre les pathologies majeures et la promotion du confinement. Globalement, l'on pourrait toujours insister sur les leviers habitats auxquels on ajoute l'accès aux services vétérinaires. La mise en œuvre des innovations proposées par le projet (construction poulaillers améliorés, fabrique d'aliment à la ferme, introduction de coqs améliorateurs) reste une nécessité. Mais cela exige une capacité financière minimale qui n'était pas le cas chez la majorité des aviculteurs ruraux. En terme d'amélioration génétique, le choix des coqs améliorateurs à vulgariser dans les fermes d'élevage, nécessite de s'assurer de leur disponibilité et de leur performance. Les croisements souvent réalisées de façon incontrôlée, sans considération des aspects de conservation de gènes et aux résultats un peu mitigés dans certains cas contribuent à l'érosion du matériel génétique de la population de poules locales. En terme socio-économique, le manque de formation technique, le faible niveau de revenu des aviculteurs villageois, l'insuffisance voire l'absence d'appui financier de la part des pouvoirs publics ne permettent pas aux éleveurs d'améliorer la productivité de leur élevage. En outre, on note les problèmes de commercialisation liés au manque d'informations sur les marchés, à l'instabilité des prix, la faiblesse des prix proposés aux éleveurs par rapport aux prix appliqués dans les centres urbains.

\section{Conclusion}

L'élevage avicole est une activité socio-économique importante qui permet aux producteurs de disposer de protéine d'origine animale de qualité mais aussi, de ressources monétaires mobilisables à tout moment. Cette étude a montré que les contraintes liées à l'habitat, aux fortes mortalités et au déficit alimentaire constituent un handicap pour le développement de l'aviculture.

C'est dans ce contexte que le projet Millenium Challenge Account en collaboration avec l'Institut Environnemental et de Recherches Agricoles (INERA) a fait un transfert de paquet technique consistant à améliorer les conditions d'élevage des volailles dans la province du Sourou. La mise en œuvre des innovations proposées par le projet a concerné la construction de poulaillers améliorés, la fabrique d'aliment à la ferme qui ont fait l'objet de test en milieu réel et en station puis l'introduction de coqs améliorateurs. 
Les travaux visaient à caractériser les systèmes de productions et à apprécier les effets d'une alimentation intensive en mode de claustration en comparaison au mode divagant en aviculture traditionnelle améliorée.

Les résultats ont montré beaucoup de variabilité en termes de caractéristiques et de performance zootechnique selon le système d'élevage pratiqué dans la zone. Les gains de poids des sujets en claustration en courte durée (3 mois) ont été importants comparés aux poulets en mode divagant, ce qui nous a permis d'apprécier la qualité nutritionnelle des aliments fabriqués et testé à la ferme. En outre, ce travail a révélé que l'introduction des coqs améliorateurs, l'amélioration de l'habitat, de la santé et de l'alimentation a amélioré les performances de croissance des volailles.

L'amélioration de l'aviculture traditionnelle dans la zone supposerait donc que la divagation diminue et qu'elle puisse évoluer vers une semi intensification. L'activité doit être soutenue et ce soutien sera d'autant plus efficace que l'Etat développera des programmes en faveur de sa promotion.

Ce travail est loin d'avoir couvert toutes les attentes et pour des investigations complémentaires nous suggérons un suivi zootechnique des volailles sur une plus longue période. En outre, l'amélioration des circuits de commercialisation permettra une augmentation régulière du nombre de volailles vendues annuellement par producteur. Il est suggéré que l'augmentation de la productivité puisse s'opérer en définissant un programme de vaccination efficace et une bonne conduite d'élevage des poussins. Ceci nécessite la mise en place d'une formation plus efficace des acteurs de la filière en vue du renforcement des capacités des aviculteurs.

\section{REFERENCES}

Anta D. 2012. Evaluation d'un transfert de paquet technique en aviculture traditionnelle et son impact sur la génération de revenu des femmes de Sibassor (KAOLACK) Sénégal.
Apuno AA, Mbap ST, Ibrahim T. 2011. Characterization of local chickens (Gallus gallusdomesticus )inShelleng and Song Local Government Areas of Adamawa State, Nigeria. Agriculture and Biology Journal of North America, 2: 6-14.

Ayssiwede SB, Deng A, Houinato MRB, Chrysostome CAAM, Missohou A.2011. Elevage des poulets traditionnels ou indigènes au Sénégal et en Afrique Subsaharienne : état des lieux et contraintes.

Benabdeljelil K, Bordas A. 2005. Prise en compte des préférences des éleveurs pour la caractérisation des populations locales de poulets au Maroc. Sixièmes Journées de la Recherche Avicole, St Malo, 30 et 31 mars 2005.

Moussa Amadou B, Idi A, Benabdeljelil K. 2010. Aviculture familiale rurale au Niger: alimentation et performances zootechniques. Publié dans Réseau International pour le Développement de l'Aviculture Familiale, 19: 2010. www.fao.org/ag/againfo/themes/en/ infpd/home.html

Bonkoungou GFX. 2005. Characteristics and performance of Guinea fowl production under improved and scavenging conditions in the Sahelian region of Burkina Faso. Master of Science Thesis. KVL - Denmark, $68 \mathrm{p}$.

CEFCOD (Centre d'Etude, de Formation et de Conseil en Développement). 2013. Situation de référence des principales filières animales au Burkina Faso. www. spcpsa.gov.bf/...ressources.../67situation-de-reference-des-princip.

Deka R, Kalifa N. 2004. Elevage de poulets de chair dans un système semi-divagant en zone rurale d'Assam (Inde). Bulletin RIDAF 14(2), Juillet - Décembre 2004.ww.fao.org/ag/againfo/subjects/en/ infpd/ home.html

Diagne A. 2012. Evaluation d'un transfert de paquet technique en aviculture traditionnelle et son impact sur la génération de revenus des femmes de 
sibassor (Kaolack) EISMV-Dakar. Thèse de Dr vétérinaire $.97 \mathrm{p}$.

DGPSE (Direction Générale de la Prévision des Statistiques de l'Elevage), 2011. Annuaire statistique Ministère des Ressources Animales. Burkina Faso. www.mra.gov.bf/annuaire $\% 20$ statistique.

El-Yuguda AD, Baba SS, Ibrahim UI, Brisibe F. 2009. Maladie de Newcastle et maladie de Gumboro chez les poulets villageois dans l'Etat de Borno (Nigeria). Bulletin RIDAF., 18(1/2) .www.fao.org/ag/againfo/subjects/en/ infpd/home.html

FAO (Food Agriculture Organization) . 2004.: Production en Aviculture Familiale.- Rome: FAO.-134p. www. fao.org/docrep/009/y5169f/y5169f00.ht $\mathrm{m}$

Fotsa JC, Bordas A, Rognon X, TixierBoichard M, Pone KD, Manjeli Y. 2007. Caractérisation des élevages et des poules locales et comparaison en station de leurs performances à celle d'une souche commerciale de type Label au Caméroun; Septièmes Journées de la Recherche Avicoles, Tours, 28 et 29 Mars 2007.

Goromela EH, Kwakkel RP, Verstegen MAW, Katule AM. 2006. Strategies to optimize the use of scavengeable feed resource base by smallholders in traditional production systems in Africa: A review. African Journal of Agricultural Research, 1(3): 091-100.

INSD. 2006. Analyse des Résultats de l'Enquête Annuelle sur les Conditions de Vie des Ménages et de Suivi de la Pauvreté en 2005. INSD : Burkina Faso. 211p.

INSD. 2007. Recensement Général de la Population et de l'Habitat 2007 du Burkina. Résultats Préliminaires. INSD Ouagadougou, Burkina Faso. 30 p. www.insd.bf/.

Kondombo SR. 2007. Importance et perspectives du secteur avicole au
Burkina Faso- FAO. www.fao.org/3/aai376f.pdf

Kouadio KE, Kreman K, Kouadja G. S, Kouao BJ, Fantodji A. 2013. Influence du système d'élevage sur la reproduction de la poule locale Gallus domesticus en Côte d'Ivoire. Journal of Applied Biosciences, 72: 5830- 5837.

Mopate. 2010. Systèmes avicoles en zone de savanes d'Afrique centrale : performances zootechniques et importance socio-économique. SeinyBoukar L, Boumard P. Savanes africaines en développement : innover pour durer, Apr 2009. Cirad: Garoua, Cameroon ; 11 p. <cirad-00472067> HAL Id: cirad-00472067.http:// hal.cirad.fr/cirad-00472067

MRA (Ministère des Ressources Animales). 2007. Diagnostic de la filière de l'aviculture traditionnelle au Burkina Faso. Rapport provisoire. Burkina Faso, $117 \mathrm{p}$.

MRA(Ministère des Ressources Animales). 2011. Contribution de l'élevage à l'économie et à la lutte contre la pauvreté, les déterminants de son développement.Burkina Faso.www. mra.gov.bf/.../etude $\% 20$ contribution $\% 20$ elevage_v\%20imprimerie.

MRAH (Ministère des Ressources Animales et Halieutiques). 2013. Journée Nationale du Paysan 16ème Edition; Banfora les 25 ,26 et 27 AVRIL. Burkina Faso.www.spcpsa.gov.bf/.../ doc.../42-document-mrah-jnp-16-2013banfora

MRA(Ministère des Ressources Animales). 2004. Deuxième enquête nationale sur les effectifs du cheptel. Tome II. Résultats et analyses. Rapport final. Ouagadougou, Burkina Faso, 77 p.

MRA(Ministère des Ressources Animales). 2006. Situation de l'Agriculture et de l'Elevage au Burkina Faso. In Ambassade de France au Burkina Faso. Fiche de Synthèse. Berger $\mathrm{P}$, Traore V (eds). MINEFI-DGTPE/AFAQ ISO 9001 : Ouagadougou, Burkina Faso. 
MRA (Ministère des Ressources Animales). 2012. Annuaire Statistiques du sous secteur de l'élevage. MRA, Burkina Faso.

Ndayisenga F. 2010. Socio-économie de l'aviculture traditionnelle dans la région de Saint- Louis (Sénégal) : Mémoire de Master II, 42p.

Moula N, Detiffe N, Farnir F, AntoineMoussiaux N, Leroy P. 2012. Aviculture familiale au Bas-Congo, République Démocratique du Congo (RDC). http://www.lrrd.org/lrrd24/5/ moul24074.htm

Ouedraogo B. 2010. Diagnostic de l'élevage avicole dans le sahel Burkinabè. Rapport de synthèse, INERA- Ouagadougou. 89 p.

PNUD (Programme des Nations Unis pour le Développement). 2006. Rapport mondial sur le développement humain 2006, New York, USA, 422 p.

Pousga B. 2009. Synthèse des travaux de recherche en aviculture au Burkina Faso: Rapport de recherche No 4. Réseau International pour le Développement de l'Aviculture Familiale, 18(1/2): 28 -35.

Sangaré. 2005. Synthèse des résultats acquis en aviculture traditionnelle dans les systèmes de productions animales
d'Afrique de l'Ouest, URPAN, PROCORDEL, CIRDES. Bobo-

Dioulasso, Burkina Faso. 66p.

Sonaiya EB, Swan SEJ. 2004. Production en Aviculture Familiale. Manuel FAO de Production et Santé Animales. Un Manuel Technique. Ed. FAO : Rome.

Soumboundou A. 2010. Evaluation de l'impact d'un transfert de paquet technique (amélioration génétique, conduite d'élevage) sur les performances zootechniques de couple mère- poussins en aviculture traditionnelle dans la zone des Niayes(Sénégal). Thèse Méd .vét, Dakar.

Traoré EH. 2006. Impact économique de la grippe aviaire (GA) sur la filière avicole sénégalaise. Communication au Séminaire international sur "Elevage et pauvreté en Afrique de l'Ouest", Mbour, mai 2006.

Traoré EH, Faye P. 2004. Rapport sur les résultats des enquêtes pré vaccination \& post vaccination contre la maladie de Newcastle (MN). Programme TCP / SEN / 2904 D FAO “Amélioration de la production avicole en élevage familial traditionnel à travers une meilleure gestion des contraintes de production et de santé animales. Phase II, août 2004. 